\title{
Comparison Between the Biologically Oriented Preparation Technique (BOPT) and the Horizontal Preparation Technique. About a Case
}

\section{Echegaray Yankova Daniel*}

Faculty of Health and Sport Sciences of Huesca, University of Zaragoza, Huesca, Aragón

*Corresponding Author: Echegaray Yankova Daniel, Faculty of Health and Sport

Sciences of Huesca, University of Zaragoza, Huesca, Aragón.
Received: May 08, 2021

Published: January 21, 2022

(C) All rights are reserved by Echegaray

Yankova Daniel.

\section{Abstract}

Introduction: One of the main complications in tooth-supported fixed dental prostheses (FDPs) is the unsatisfactory aesthetic result due to the apical migration of the gingival margin. The tendency of the gingival margin to migrate apically over time is related to different factors (gingival biotype, prosthetic preparation, invasion of biological width, iatrogeny, etc.). Within them, one is particularly relevant, the preparation technique and the corresponding geometry of the finishing line. The placement and design of the finishing line for dental preparation has always been a topic of controversy in the literature. The debate is whether horizontal preparation is superior to vertical preparation and viceversa in terms of ease of manufacture, tissue stability and long-term survival, among others.

The biologically oriented preparation technique, or BOPT, arises as a preparation technique in which periodontics and prosthesis will work together. What is intended with this technique is to guide the tissues periodontally through prosthetic rehabilitation in order to eliminate or minimize the apical migration of the gingival margin.

Objectives: To compare the BOPT technique with a preparation technique with horizontal finishing line through the realization of two FDPs, evaluating the clinical and biological aspects at one year of follow-up.

Material and Methods: A 48-year-old patient comes to the clinic whose reason for consultation is to replace the absence of the upper second premolars. Given the refusal to implants, the treatment plan offered is the realization of two FDPs. For the replacement of the 1.5 a BOPT preparation is made, while for the replacement of the 2.5 a juxta gingival preparation is made.

Results: In the case of the BOPT technique, better tissue stability and increased gingival thickness are observed. In the case of the horizontal technique, a slight apical migration of the gingival margin is observed in two locations, in addition to bleeding at probing in four of twelve locations.

Conclusion: Different designs of finishing lines have been indicated in dental preparations for different reasons, but it is unclear which type of finishing line, if any, offers the greatest advantages. Apparently the BOPT technique is the one that obtains the best results. However, to give greater scientific value to the technique, more clinical and biological studies are needed.

Keywords: Prosthetic Finishing Line; Recession; BOPT Technique; Vertical Preparation; Horizontal preparation; Chámfer; Fixed Prosthesis

\section{Introduction}

One of the main complications in tooth-supported fixed dental prostheses (FDPs) is the unsatisfactory aesthetic result due to api- cal migration of the gingival margin. The tendency of the gingival margin to migrate apically over time is related to different factors (inadequate amount and quality of keratinized tissue, reaction to 
trauma during prosthetic work, chronic inflammation due to prosthetic errors, trauma due to inadequate brushing, etc.). Among them, one is particularly relevant, the preparation technique and the corresponding geometry of the finishing line [1].

The The biologically oriented preparation technique (BOPT), introduced in 2008 by Ignazio Loi, is inspired by the studies carried out by Carnevale and Di Febo, who in the 1990s adopted a prosthetic clinical protocol for highly periodontally compromised cases. This protocol consisted of preparing the teeth, after raising a flap, up to the bone crest. The objective was to eliminate undercuts, facilitate dental preparation and reduce the concavity of the roots. The tissues were allowed to heal and, between 8 and 12 weeks after surgery, prosthetic rehabilitation was performed. With this technique, a remodeling of the dentogingival complex was achieved. The main difference with the BOPT technique is that the latter can be used indistinctly on all teeth (periodontal or not) and does not require flaps (absence of surgery) [2-4].

The BOPT technique, used by Dr. Ignazio Loi for more than 15 years, has proven successful in maintaining pericoronal soft tissue stability in both anterior and posterior areas. With the BOPT technique, the clinician and laboratory technician can interact with surrounding tissues by modifying their shape and scalloped architecture, regardless of any pre-existing dental or gingival limitations [1]. The advantages are relevant considering that most of the clinical results are obtained only through the restoration, both provisional and final (margin position, emergency profile, tooth shape).

This article presents a clinical case in which two FDPs are performed, comparing a preparation technique using a horizontal finishing line (juxtagingival chamfer) and the BOPT technique, at one year of follow-up.

\section{Material and Methods}

A 48 year old women came to the dental practices service of the Faculty of Health and Sports Sciences of Huesca (University of Zaragoza) whose reason for consultation was to replace the absence of the upper second premolars. In the intraoral examination, no anomaly was detected, and the periodontal status was correct (physiological probing, absence of bleeding, absence of mobility). After the intraoral examination, the treatment plan offered is the performance of two FDPs, since for economic reasons the option of implants is rejected. For the replacement of the 1.5, a BOPT technique is carried out, while for the replacement of the 2.5 a horizontal preparation (chamfer) is made.
In the case of the horizontal preparation, with a chamfer diamond bur of 100-200 $\mu \mathrm{m}$ and $1.4 \mathrm{~mm}$ in diameter (ADO-881, G014; Ancladén SL) the occlusal reduction is started, preserving the shape basic geometry. After that, the axial reduction is performed, tracing guide grooves. Subsequently, the juxtagingival chamfer termination line is marked and the parallelism between abutments is checked. Finally, the fit of the metal is checked, the biscuit try-in is done, and the final prosthesis is placed. (Figure 1).

In the case of the BOPT technique, a double probing is initially performed, first to the sulcus to measure the depth of the gingival

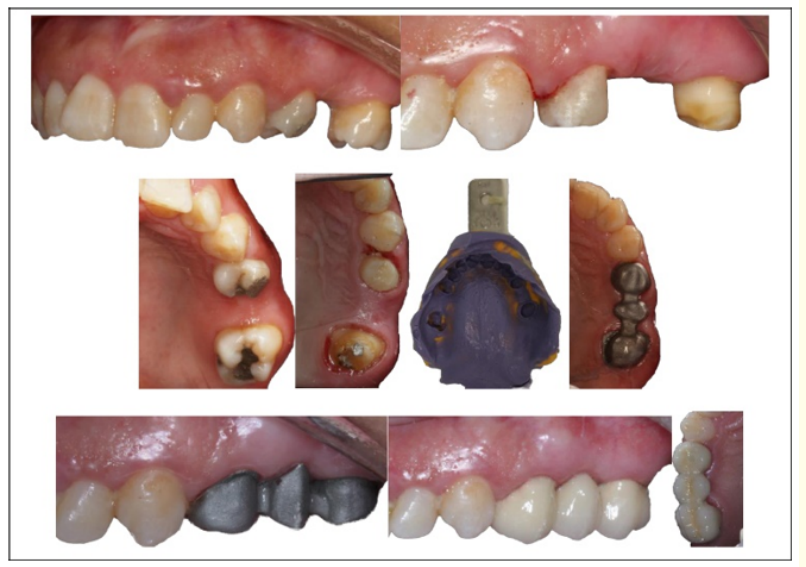

Figure 1: Horizontal preparation technique (juxtagingival chamfer).

sulcus and then to the bone crest, to measure the bone level and locate the cementoenamel junction (CEJ), since this determines the limit of the preparation.

Tooth preparation begins by reducing the incisal edge by $2 \mathrm{~mm}$. The axial walls are reduced $1 \mathrm{~mm}$ with a diamond bur. Then, the inner wall of the sulcus and the tooth are prepared at the same time with a conical diamond bur (BOPT bur 862.514.012; Sweden \& Martina) of $1.2 \mathrm{~mm}$ diameter. The bur is inserted into the sulcus at an angle of 15 degrees. The goal of this is to remove $1 \mathrm{~mm}$ from emergence of the anatomical crown. Next, to avoid creating a finishing line, the bur is positioned parallel to the axis of the tooth between the root and the crown, thereby removing tooth structure with the body of the instrument rather than the tip. Both the tooth and the gum are prepared at the same time. After that, the provisional prosthesis is relined and adapted to the abutment using a self-curing acrylic resin (Sintodent White; Sintodent). With the provisional relined, 2 areas are distinguished: an internal one that 
determines the extension of the gingival sulcus and an external one that marks the position of the gingival margin. Between these areas, a groove is formed in the provisional prosthesis, which is filled with light-curing flowable resin. This union will create the cervical emergence profile of the provisional restoration. Using the provisionals, a CEJ is created with the new emergence profile. Provisional restorations are placed at a depth of 0.5 to $1 \mathrm{~mm}$ in the sulcus, respecting the biological width. After a period of 4 weeks, the provisional is lifted and it is verified that the biological space has been invaded in two locations. When the biological width is invaded, the only structure that has a biological reaction is the connective tissue, which induces an inflammatory reaction that leads to the production of inflammatory molecules that will activate the osteoclasts, inducing bone resorption and risk of soft tissue recession. Given this, a provisional modification is made, correcting the involuntary vertical overextension. At 8 weeks, with the soft tissues showing a healthy appearance, impressions are taken. A 2-step impression technique is used with elastomers (Ivoclar Vivadent AG) and 2 gingival retraction cords (Ultrapack; Ultradent Products Inc.). The definitive prosthesis is created based on the biological and functional parameters of the provisional restorations. Once placed, aesthetics, marginal fit, interproximal contacts and occlusion are evaluated. (Figure 2)

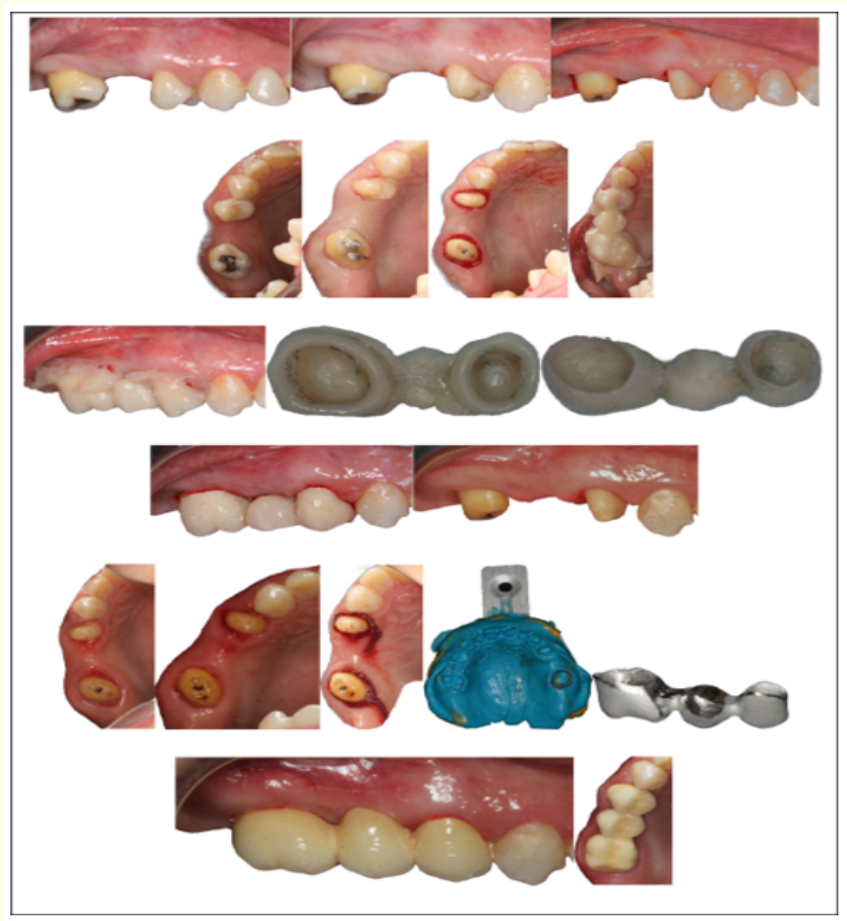

Figure 2: BOPT technique.

\section{Discussion}

Tooth-supported fixed dental prostheses treatment (FDPs) continues to be one of the main options when it comes to replacing missing teeth. One of the most feared phenomena in these treatments is the instability of the gingival tissue, which may be associated with periodontal inflammation resulting from the injury of the prosthesis or with the apical migration of the gingival margin around the restoration. This implies the exposure of the interface between tooth and prosthesis which, in most cases and especially in areas of aesthetic relevance, is extremely unpleasant and leads to the failure of the prosthesis. Among the factors related to this (gingival biotype, trauma, iatrogenia, etc.) it has been observed that the design or geometry of the preparation and the location of the finishing line play a fundamental role in gingival health. If the interface fit is not well adapted (marginal inaccuracy) it will be a biological refuge for periodontopathogenic bacteria, allowing them to damage the dental support structures and cause the failure of the prosthesis [4-12].

Over the years the realization of FDPs has been carried out through the use of finishing lines in the dental preparation. The finishing line is described as the border between the intact portion of the tooth and the most apical point of the preparation. It must be well defined, regular and, above all, well positioned. There are many finishing lines and the use of one or another depends on the treatment plan, trends, and the type of material used (metal, ceramic, metal-ceramic, lithium disilicate or zirconium, among others). In practical conditions, the factor that most influences the design is the restorative material. It also influences the anatomy and position of the teeth, the number of teeth involved and the skill, precision and experience of the operator [5, 9,13].

The location and design of the finishing line has been, and continues to be, a topic of great debate and importance among numerous authors. Their objective is to establish the type of preparation with the best long-term prognosis $[3,11]$.

Depending on the location, we find supragingival lines (preparation coronal to the gingival margin), juxtagingival lines (preparation at the same level as the gingival margin) or subgingival lines (preparation apical to the gingival margin) [13].

Based on the design, Kuwata [14] established a finishing line classification from the point of view of the marginal angle (vertical projection from the outer surface of the tooth and its prepared surface). He defined a marginal angle between 0 and $30^{\circ}$ as a bevel, between 31 and $60^{\circ}$ as a chamfer, and between 61 and $90^{\circ}$ as a 
shoulder. Later, a more practical classification was proposed, distinguishing between horizontal finishing lines and vertical finishing lines. Within the horizontal ones are the chamfer, chamfer with bevel, shoulder, shoulder with bevel and sloped shoulder, and within the vertical ones the knife edge and the feather edge or softened preparation. Some authors include the BOPT technique within this last group while others indicate that it is a preparation without a termination line $[1,2,6,8,9,12]$.

The vertical preparation technique or without finishing line is described by Morton Amsterdam since 1968 and later by Carnevale and Di Febo. Over the years, in cases of periodontal affected teeth, vertical preparation was the only recourse, as it was impossible to prepare a shoulder or chamfer at root level. However, over time it was used less, being replaced by techniques with finishing margins $[3,12,15]$.

Horizontal preparations have a very obvious line and are recommended in cases where the periodontium is healthy and the clinical crown matches with the anatomical one. In them, the prosthetic margins are located near the cementoenamel junction (CEJ). Vertical preparations or without finishing line are more conservative and recommended when the clinical crown, due to a loss of periodontal support, does not match with the anatomical crown. In these cases, the prosthetic margin is placed in the root area. Based on this, some authors state that the termination line depends more on a clinical factor than on a technical factor $[1,6,9,10]$.

Horizontal preparations can be identified, are easily visible, have a defined margin and, according to their advocates, allow for better seating of the restoration. In them, the margin is positioned by the dentist and a well-defined finishing line is left that will be transmitted to the impression and the working model. This is probably the reason why prosthodontists prefer horizontal preparations. In the BOPT technique, the margin is positioned by the laboratory technician based on information from the gingival tissue. A fundamental concept is that the finishing line of the horizontal preparations is in the prepared tooth, so that in the laboratory the technician only has that information to make the prosthesis on the tooth. On the other hand, in the BOPT technique, the finishing line is the margin of the prosthetic crown. The emerging anatomy of the tooth is eliminated and inclined planes without a finishing line are created, which will allow the laboratory technician to have a finishing area in which he will have absolute freedom to position the margin of the prosthesis. This margin may be shortened or extended both in the temporary and in the final restoration at different intrasulcular levels, without invading the gingival sulcus (controlled invasion of the sulcus) $[9,16]$.

Many studies claim that tooth preparation facilitates placement of the prosthetic restoration, reducing marginal discrepancy. One of the greatest difficulties for the clinician is to place the prosthetic margin in an apicocoronal direction in a clear, precise and biologically correct manner. The concept of subgingival positioning of the margins of a prosthetic crown is based on aesthetic reasons, although several authors believe juxta or supragingival placement is more appropriate for the maintenance of a healthy periodontium in the long term $[2,6,7,8,17]$. Various studies have observed that subgingival restorations with a horizontal finishing line are associated with periodontal inflammation (bleeding four to five times higher than in supragingival margins) and possible gingival recession [18-24]. This is due to the inability to establish an adequate marginal fit in the subgingival region. In the case of vertical finishing line subgingival restorations, many in vitro and in vivo studies have shown that the vertical preparation has the least marginal discrepancy compared to other preparation designs. Therefore, it can be said that the coronal seal is definitely better in vertical preparations than in horizontal preparations. This is due to the decreased space between the teeth and the crown as a result of the vertical geometry. The result is a better fit of the crown, less exposure of the root cement, less extrusion of cement during the cementation phase and less bacterial penetration. Some authors have also shown that a poor periodontal response depends more on the poor adaptation of the crown margin than on the placement of the final margin within the gingival sulcus. Thus, it has been observed that, despite the subgingival position of the prosthetic margin, the soft tissues appear to be healthy and stable [1, 6, 9, 10, 12, 17, 25].

Poor periodontal response has also been associated with the shape of the crown contour. Crown contours obtained with the BOPT technique may appear excessively pronounced, according to the traditional definition of "overcontouring". Overcontouring has frequently been considered a deleterious anomaly in crown construction, leading to tissue inflammation and periodontal problems [23]. The crown contour has two main components, the emergence profile and the cervical contour. The term emergence profile 
is defined as "the contour of a tooth or restoration in relation to the adjacent tissues". For its part, the cervical contour refers to the curvature that must always be recreated in artificial crowns, it is the subgingival cervical beginning. Its function is to maintain the surrounding tissues in tension and health. The cervical contour corresponds to the CEJ and is neither flat nor concave, but convex. The amount of this convexity can be measured through the angle of emergence. When removing the CEJ, this anatomical landmark must be artificially recreated with a physiological angle that supports the surrounding soft tissue. This should not be seen as an overcontour but rather as a new contour or a new artificially recreated CEJ [12]. In addition, Loi et al [1] are of the opinion that the concept of overcontouring should be reinterpreted. In fact, there is no consensus on what a "normal" contour should be. Sorensen suggested that a vertical contour of up to 45 degrees can still be considered normal. In contrast to what other authors suggest, in most cases of BOPT it is very rare to observe a swollen gingiva and a recession related to the contours of the crown [1]. Moreover, with a physiological overcontour, biologically conformed, with a harmonious emergence and a controlled gingival compression, no harmful effect of gingivitis or recession is caused, but rather the opposite, since it allows to increase the magnitude of sealing of the gum barrier [10].

The stability of the gingival tissues and the harmony between the tooth and the gum represent a challenge for prosthetic dentistry. In fact, the most difficult variable to predict is the gingival response to the prosthetic restoration. A correct relationship between dental restorations and periodontal tissues is extremely important to ensure long-term success, as well as aesthetics. If, on the one hand, the periodontium must be in good condition so that the rehabilitation remains in optimal conditions for a long period, on the other hand, the prosthetic rehabilitation must show adaptation to the periodontal tissues so that they can remain healthy. In the past, prosthetic techniques were used that affected the gingival component as little as possible, although, nowadays, it has been observed that, compared to conventional techniques, BOPT is capable of promoting short, medium and long-term soft tissue stability, adapting by themselves and naturally to the preparation and restoration. Once the convex anatomy of the tooth is transferred to the final prosthesis, what is sought is for the gum to thicken and adapt around the new shapes and profiles created. In this technique, prosthetic and periodontium merged into a new concept of emerging profiles or adaptation profiles, where the technician has an active participation in the placement of the finishing line and in the choice of prosthetic profiles, being a restorative protocol conceived to reproduce dental nature. With BOPT it is possible to correct recessions and thicken soft tissues $[1,5,9,10,11]$.

In summary, the advantages and disadvantages of both techniques are:

Advantages of horizontal termination lines [2,15]

- $\quad$ Easy preparation.

- Well-defined and visible margins.

- $\quad$ Ease of work between clinician and laboratory.

- Greater precision during the construction phases.

- Absence of overcontouring.

- In metal-ceramic restorations, they provide sufficient rigidity so that the porcelain firing does not distort the metal margin of the restoration.

\section{Disadvantages of horizontal preparations}

- Less marginal fit: increased risk of caries, pulpitis, necrosis, fracture of the restored tooth, gingivitis and recession.

- Less tissue stability: risk of recession and, consequently, aesthetic failure.

- Impossibility of making two identical finishing lines.

Advantages of BOPT technique [1, 7, 9, 4, 16, 26-31]

- CEJ removal on unprepared teeth and removal of existing finishing lines on already prepared teeth (useful in retreatments).

- $\quad$ Possibility of placing the finishing line at different levels, either more coronally or more apically, within the gingival sulcus (controlled invasion of the sulcus), without affecting the quality of marginal adaptation of the restoration.

- Possibility of realization both in periodontal teeth and not.

- Possibility of modulating the crown emergence profiles to create the ideal esthetic gingival architecture.

- Creation of a new CEJ through the prosthesis.

- More conservative procedure: saving tooth structure.

- $\quad$ Ease and speed of execution.

- Optimum restoration-tooth relationship.

- $\quad$ Ease of finishing and relining temporary crowns. 
- $\quad$ Ease of impression taking: a defined finishing line does not have to be reproduced, but rather the opening of the gingival sulcus.

- Increased gingival thickness.

- Possibility of leveling gingival margins without the need for surgery.

- Greater stability of the gingival margin in the short, medium and long term.

- Better marginal fit: greater retention and better fit between restoration and tooth.

- $\quad$ They are the best clinical solution in cases of advanced periodontal damage that require a fixed prosthesis when the crown margin is located in the root area.

Disadvantages of BOPT technique $[2,4,7-9,15]$

- According to its detractors: overcontouring, uncontrolled invasion of the gingival sulcus, excessive taper of the prepared tooth, distortion of the ceramic during firing, profuse bleeding, unpredictable soft tissue regeneration, absence of a sharp, well-defined or visible finishing line, and difficulty in clinical and laboratory work.

- $\quad$ More time in the dental chair.

- Minimum waiting period of 4 weeks.

- Fracture or debonding of provisionals during the healing period.

- High learning curve: requires exceptional preparation skills.

- Difficulty in placing the prosthetic margin adequately as there is no finishing line to refer to.

- After cementation, in cases of material excess, removal is complex due to the subgingival location of the restoration's emergence profile.

- Difficulty in performing adhesive cementation (impossibility of isolating the field).

- For the clinician or laboratory technician with little experience in the procedure, there is a risk of uncontrolled sulcus invasion.

- $\quad$ Need for more scientific evidence..
In this clinical case, a comparison is made between a horizontal finishing line (chamfer) and the BOPT technique. In the preparation with a finishing line, greater complexity is observed making and marking the chamfer and when taking the impression. In the BOPT technique, preparation, is less complex, the complexity of the technique being based on the conformation of the provisional. The conformation of a provisional with a correct emergence profile and controlling the invasion of the sulcus entails a longer time in the dental chair. In the conventional technique, the final impression is taken in the same session in which the preparation is performed, while in the BOPT technique it is necessary to wait a minimum period of 4 weeks of provisionalization for the soft tissues to mature, being able to take the final impression after 4 weeks. The BOPT technique necessarily requires a provisional fabricated by the laboratory, increasing the expense of the treatment. In both cases, the prosthesis is cemented during the first 2 months with provisional cement and, after that time, it is permanently cemented. Follow-up is 1 year in both cases. In the case of the conventional technique, over the course of a year, a slight apical migration of the gingival margin can be seen at the level of the mesiobuccal surface of 2.4 and the mesiopalatine surface of 2.6. (Figure 3). In the follow-up of the BOPT technique, tissue stability and slight thickening of the gingival tissues can be seen. This finding agrees with those of the study by Serra et al [26], where it is observed that gingival thickening is more evident during the first year. In the same study it is also observed that the long-term stability of soft tissues is $98.6 \%$. In the period of observation of the BOPT technique, no inflammation or bleeding on probing is observed. In the medium term, Paniz et al [18], observed bleeding of $41.3 \%$. Pettinicchio et al [17], observed that inflammation was greater in BOPT preparations than in those performed with the shoulder. This differs from the long-term results obtained by Serra et al [26], who observed bleeding of $12 \%$ at 4 years. Thus, in contrast to what other authors suggest, in most BOPT cases it is very rare to observe inflammation or recession associated with the contours of the crown $[1,9]$. In the case of the conventional technique, bleeding is observed in 5 locations, which agrees with the studies by Pelaez et al [20] and Tanner et al [32], where a high percentage of inflammation and bleeding is observed in the long term in preparations made with chamfer. 


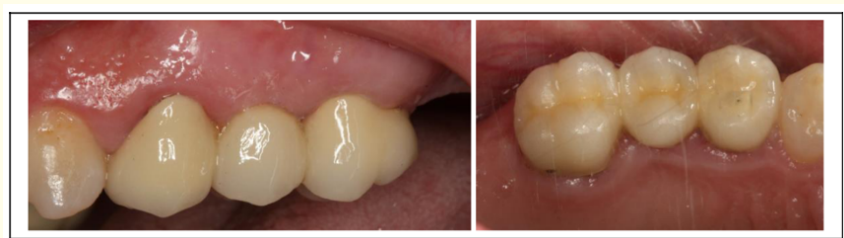

Figure 3: Slight recession at the level of $2.4(\mathrm{MV})$ and $2.6(\mathrm{MP})$.

\section{Conclusions}

- $\quad$ The BOPT technique has proven to be effective in increasing gingival thickness, providing stability to the gingival margin over time.

- $\quad$ The BOPT technique is capable of producing a coronal migration of the gingival margin.

- Different finish line designs have been suggested for tooth preparations for different reasons, but it is not clear which type of finishing line, if any, offers the greatest advantage. Apparently the BOPT technique is the one that obtains the best results. However, to give greater scientific value to the technique, more clinical and biological studies are necessary.

\section{Bibliography}

1. Loi I and Di Felice A. "Biologically oriented preparation technique (BOPT): a new approach for prosthetic restoration of periodontically healthy teeth". The International Journal of Esthetic Dentistry 8.1 (2013): 10-23.

2. Loi I., et al. "Biologically oriented preparation technique (BOPT). A new approach in prosthetic preparation in dentistry". International Quintessence 5 (2008): 69-75.

3. Bazzoli M., et al. "The management of gum parabolas with B.O.P.T. technique approach with cad-cam". Teamwork Media 9.5 (2013): 42-53.

4. Agustín-Panadero R and Solá-Ruíz MF. "Vertical preparation for fixed prosthesis rehabilitation in the anterior sector". Journal of Prosthetic Dentistry 4 (2015): 474-478.

5. Viviani A., et al. "Description of the BOPT (Biological Oriented Preparation Technique)”. Dental Gazette 298 (2018): 134-147.
6. Loi I., et al. "The prosthetic coronal contour with biologically oriented preparation technique (BOPT): technical considerations". Quintessence International 25 (2009): 4-19.

7. Agustín-Panadero., et al. "Fixed dental prostheses with vertical tooth preparations without finish lines: A report of two patients". The Journal of Prosthetic Dentistry 115.5 (2016): 520-526.

8. Agustín-Panadero R., et al. "Prospective Clinical Study of Zirconia Full-coverage Restorations on Teeth Prepared with Biologically Oriented Preparation Technique on Gingival Health: Results After Two-year Follow-up". Operative Dentistry 43.5 (2018): 482-487.

9. CWC Oaks. "B.O.P.T. Biologically Oriented Preparation Technique" (2017).

10. Castorani C., et al. "Correction of gingival parables with biologically oriented preparation technique". Dental Cadmos 83.6 (2015): 425-434.

11. Loi I., et al. "The influence of dental preparation on the gingival margin. Management of periodonto with biologically oriented technique (BOPT) in a case of deep dental fractures". Clinical Periodontics 5.12 (2019): 37-46.

12. Scutellà F., et al. "A Retrospective Periodontal Assessment of 137 Teeth After Featheredge Preparation and Gingittage". International Journal of Periodontics and Restorative Dentistry 37.6 (2017): 791-800.

13. Baker RA and López CC. "Clinical-prosthetic protocol of the BOPT technique. Barcelona: European Specialized Editions (2016).

14. Kuwata M. "Gingival margin design of abutments for ceramometal restorations. 2". Quintessence of Dental Technology 10 (1979): 27-38.

15. Magallanes R., et al. "The Shoulderless Approach a New Rationale in Prosthetic Dentistry". Tomorrow Tooth Journal 1 (2017): 1-29.

16. Castorani C., et al. "Valutazione clinica della risposta dei tessuti gengivali alla tecnica BOPT". Il Dentista Moderno 10 (2017): 58-66. 
17. Pettinicchio M., et al. "Clinical and histological outcomes of subgingival knife-edge tooth preparation". Case Reports. Dental Cadmos 79.7 (2011): 420-429.

18. Paniz G., et al. "Periodontal response to two different subgingival restorative margin designs: a 12-month randomized clinical trial”. Clinical Oral Investigations 20.6 (2016): 1243-1252.

19. Paniz G., et al. "Clinical periodontal response to anterior all-ceramic crowns with either chamfer or feather edge subgingival tooth preparations: six-month results and patient perception". International Journal of Periodontics and Restorative Dentistry 37 (2017): 61-68.

20. Pelaez J., et al. "A four-year prospective clinical evaluation of zirconia and metal-ceramic posterior fixed dental prostheses". International Journal of Prosthodontics 25 (2012): 451-458.

21. Moretti LA., et al. "The influence of restorations and prosthetic crowns finishing lines on inflammatory levels after non-surgical periodontal therapy". The Journal of International Academy of Periodontology 13.3 (2011): 65-72.

22. Ozcan M., et al. "Influence of cervical finish line type on the marginal adaptation of zirconia ceramic crowns". Operative Dentistry 34 (5 (2009): 586-592.

23. Orkin DA., et al. "The relationship of the position of crown margins to gingival health". Journal of Prosthetic Dentistry 57.4 (1987): 421-425.

24. Silness J. "Periodontal conditions in patients treated with dental bridges. 3. The relationship between the location of the crown margin and the periodontal condition". Journal of Periodontal Research 5 (1970): 225-229.

25. Richter WA and Ueno H. "Relationship of crown margin placement to gingival inflammation". Journal of Prosthetic Dentistry 30 (2 (1973): 156-161.

26. Serra-Pastor B., et al. "Periodontal and prosthetic outcomes on teeth prepared with biologically oriented preparation technique: a 4-year follow-up prospective clinical study". Journal of Periodontal Research 63.4 (2019).

27. Patroni S., et al. "CAD/CAM technology and zirconium oxide with feather-edge marginal preparation". The European Journal of Esthetic Dentistry 5 (2010): 78-100.

28. Poggio CE., et al. "A retrospective analysis of 102 zirconia single crowns with knife-edge margins". Journal of Prosthetic Dentistry 107 (2012): 316-321.
29. Schmitz JH., et al. "Monolithic lithium disilicate complete single crowns with feather-edge preparation design in the posterior region: a multicentric retrospective study up to 12 years". Quintessence International 20 (2017): 601-608.

30. Tacchini L and Bazzoli M. "Class $\mathrm{V}$ conservative restorations according to the principles of BOPT technique: the tissue responses. Case report with a 40-month follow-up". Il Dentista Moderno 11 (2017): 62-70.

31. Agustín-Panadero R., et al. "Dental-gingival remodelling with BOPT no-prep veneers". Journal of Clinical and Experimental Dentistry 9.12 (2017): 1496-1500.

32. Tanner J., et al. "Zirconia single crowns and multiple-unit FDPs-an up to 8 -year retrospective clinical study". Journal of Dentistry 79 (2018): 96-101.

\section{Assets from publication with us}

- Prompt Acknowledgement after receiving the article

- Thorough Double blinded peer review

- Rapid Publication

- Issue of Publication Certificate

- High visibility of your Published work

Website: www.actascientific.com/

Submit Article: www.actascientific.com/submission.php

Email us: editor@actascientific.com

Contact us: +91 9182824667 